of myoglobin in the late 1950s, for which they shared the Nobel Prize in Chemistry in 1962. Piccole Visioni extends these developments with an account of the subsequent higher-resolution structures of deoxygenated and oxygenated haemoglobin.

Piccole Visioni offers a lively and penetrating insight into the life and work of Perutz. Paterlini writes clearly and her book is well researched, successfully portraying both Perutz's science and his humanity, honesty and ability to reach a decision by persuasion. It has been argued that these qualities were instrumental in developing the MRC Laboratory of Molecular Biology into a highly original and successful research institution - indeed, Perutz's style of management survived his retirement as chairman of the laboratory in 1979. Perutz also played an early and important part in the genesis of the European Molecular Biology Organisation (EMBO).

Piccole Visioni does not deal with another of Perutz's legacies - namely, his writings for the public, now a commonplace initiative. $\mathrm{He}$ wrote several beautiful short essays collected in books such as Is Science Necessary? (1989), Science is Not a Quiet Life (1997) and I Wish I'd Made You Angry Earlier (1998, 2002).

Piccole Visioni is among the first of several books that address Max Perutz's gigantic contribution to twentieth-century biology and science culture. Paterlini sets the scene for these and her book will hold its place among them. I hope that it will soon become available in other languages.

Ermanno Gherardi is at the Medical Research Council Centre, Cambridge CB2 2QH, UK.

\title{
Heaven in grains of sand
}

\section{Nanoscientists and Tibetan monks unite to explore the mysteries of the mandala.}

\section{Martin Kemp}

Western religious art from the time of ancient Greece has generally relied on the representation of the human figure. But in many world cultures, especially those that proscribe the literal depiction of any deity, symbolic schemata and patterns have been used to express the truths of spiritual life.

Over the centuries, such patterns have evolved extraordinary levels of intricacy. Islamic tiling, famed for its remarkable mathematical symmetries, provides the most commonly cited example of age-old pattern generation that continues to engage our intellectual and aesthetic interests.

Less familiar, but no less striking, are the many Buddhist variations of the mandala, which symbolizes the Universe. The outer region of the mandala - 'that which encircles' - expresses the cosmological world system, whereas the inner zones characteristically progress to the realm of the highest deities. Dense, with map-like features, from grand structures to tiny symbolic details, mandalas serve the spiritual exercise of diligently sustained contemplation.

The most astonishing are the Tibetan mandalas composed from coloured grains of loose sand (www.imagesandmusic. $\mathrm{nl} / \mathrm{dhtml} /$ Mandala.htm\#). The time and patience required to create them is incredible to anyone involved in the frenzy of modern life. The meticulous, laborious act of making it is itself a key part of the discipline of timeless meditation. It is, as William Blake wrote, "to see heaven in a grain of sand".

This compound of extreme physica minuteness and grand cosmological structure has recently provided the rationale for the presence of a group of Tibetan monks in a leading nanotechnology laboratory. The initiative is led jointly by an artist and a chemist at the University of California in Los Angeles - Victoria Vesna from the Department of Design | Media Arts, and nanoscientist James Gimzewski from the Department of Chemistry and Biochemistry

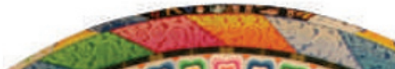

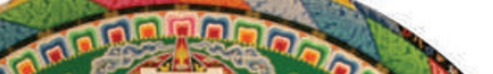

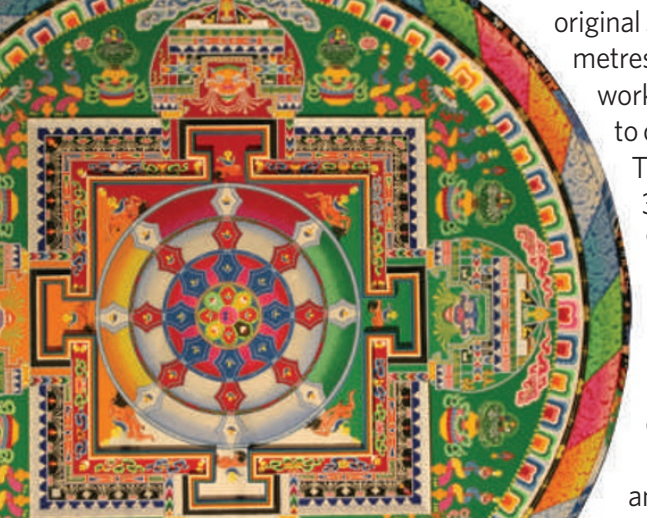
nonk

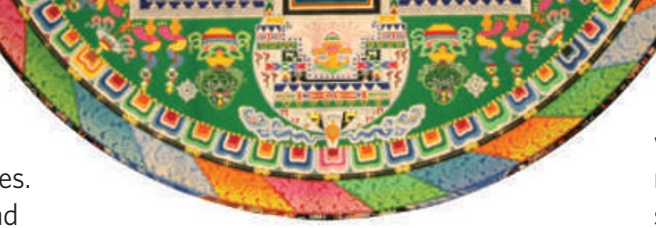

The Chakrasamvara Mandala is made of coloured sand. Its intricacies have been revealed down to the molecular level.

(see http://nano.arts.ucla.edu/mandala).

They have collaborated with monks from the Ghaden Lhopa Khangsten Monastery in India to explore a Chakrasamvara mandala. The mandala, called the 'wheel of great bliss', encircles the palatial residence of the deity Heruka Chakrasamvara. It places particular emphasis on the female ideal of wisdom.

As an extension of the monks' rendering of the cosmological whole from the tiniest grains, Gimzewski has used optical and scanning electron microscopy to delve into progressively smaller features of the sand mandala, right down to the molecular level. Microscopic images across this range were then blended with a sequence of zoomed photographs to produce a continuous visual journey from the whole mandala into everfiner details of its physical composition. The result is a seamless 15 -minute sequence that is projected onto a circular bed of flat, unpatterned sand.

The numbers involved are awesome. The original sand mandala was two-and-a-half metres in diameter and took four monks working for eight hours a day four weeks to complete in Gimzewski's laboratory. The final computer output comprised 30,000 individual frames containing 900 gigabytes of data. Thirty-six computers were pressed into service to render the images over the course of two days, and nine computers completed the recomposition of the continuous sequence.

There is something very beautiful and moving in this holy alliance of Buddhist spiritual patience, founded on minute care and untiring repetition, and the unholy processes of iteration of which modern computers are capable. The mandala-makers and the nanoscientists share the wonder of scale, involving countless parts to compose the ordered whole. We can sense the way in which religious contemplation of a time-honoured kind and modern technological science are, in their different ways, reaching out to the edges of infinity.

This is the aesthetic realm of the sublime. It is inhabited by all those who stand in awe at the wonder of the Universe and in thrall to the varied mental capacities we use to make sense of what we see and feel.

Martin Kemp is professor of the history of art at the University of Oxford, Oxford OX1 IPT, UK.

\footnotetext{
The piece is to be exhibited at the Maison Européenne de la Photographie in Paris until 30 September then at the Singapore Science Centre until 8 December 2011.
} 